\title{
Síndrome del cóndilo occipital como primera manifestación de mieloma múltiple. Reporte de caso y revisión de la literatura
}

\author{
Occipital condyle syndrome as the presentation of multiple \\ myeloma. Case report and literature review
}

\author{
Jeferson Arango-Rodríguez', David Vergara-García ${ }^{1}$, Andrea Catalina Sandoval', Fernando Portilla ${ }^{1}$ \\ ${ }^{1}$ Departamento de Neurocirugía, Centro de Investigación y Entrenamiento en Neurocirugía (CIEN), Hospital Universitario de la \\ Samaritana, Escuela de Medicina Universidad del Rosario. Bogotá, Colombia.
}

\begin{abstract}
Resumen
El síndrome del cóndilo occipital es un conjunto de síntomas que incluyen dolor occipital unilateral asociado a paresia del XII par craneal ipsilateral. Es poco común y generalmente está asociado a lesiones tumorales metastásicas, más frecuentemente de cáncer de próstata y cáncer de mama. En este artículo se presenta el caso de una paciente femenina previamente sana con síntomas clásicos de síndrome de cóndilo occipital izquierdo, en quien estudios de tomografía y resonancia documentaron lesión lítica que comprometía el cóndilo occipital izquierdo. Por la presencia de signos de inestabilidad ósea cráneo cervical y la ausencia de diagnóstico histopatológico, se decidió llevar a resección de lesión y artrodesis occipitocervical por medio de abordaje extremo lateral para cóndilo occipital izquierdo. Los estudios histopatológicos de la lesión dictaminaron infiltración ósea por mieloma múltiple.
\end{abstract}

Palabras clave: Cóndilo, metástasis, mieloma múltiple, par craneal, semiología.

\section{Abstract}

The occipital condyle syndrome presents with unilateral occipital pain associated with paresis of the ipsilateral XII cranial nerve. It is uncommon and is usually associated with metastatic tumor lesions, most frequently prostate and breast cancer. In this article we present the case of a previously healthy female patient with classic symptoms of left occipital condyle syndrome. Computed tomography and magnetic resonance imaging studies documented a lytic lesion that compromised the left occipital condyle. Due to the presence of signs of craneocervical instability and the absence of histopathological diagnosis, we decided to take the patient to resection of the lesion and occipitocervical arthrodesis. We used a far-lateral approach to the left occipital condyle. Histopathological study of the lesion confirmed bone infiltration by multiple myeloma.

Key words: Condyle, metastasis, multiple myeloma, cranial nerves, semiology.

\section{Correspondencia a:}

David Vergara-García, MD.

Centro de Investigación y Entrenamiento en Neurocirugía (CIEN), Departamento de Neurocirugía, Hospital Universitario de la Samaritana, Departamento de Neurocirugía, Escuela de Medicina, Universidad del Rosario.

Carrera 8 No. 0-29 Sur, Bogotá DC.

Teléfono: (+571) 4077075 Ext 10472

david.vergara@urosario.edu.co 


\section{Introducción}

El síndrome del cóndilo occipital consiste en la presencia de dolor en región occipital unilateral, que empeora con la rotación de la cabeza hacia el lado contrario, y paresia del XII par craneal ipsilateral, lo cual genera dificultad para la articulación de palabras y/o dificultad para $\operatorname{tragar}^{1,2,3,4,5,6,7,8,9,10,16,17,19,20}$. Se debe al compromiso del conducto del hipogloso por el cual emerge el XII par craneal. Su principal etiología es la enfermedad neoplásica secundaria $^{3,5,6,7,9,10,19,20)}$. Las lesiones primarias más comúnmente asociadas son el cáncer de mama y de próstata, seguidos por los tumores colorrectales, renales, nasofaríngeos, óseos primarios y por ultimo linfomas $2,7,8,11,12,13,16,17$. Otras enfermedades que también afectan el conducto del hipogloso en el cóndilo occipital son traumas, enfermedades inflamatorias y lesiones post-radiación ${ }^{15,19,20}$. Por su cercanía, puede encontrarse afección concomitante de los pares craneales IX A XI en el foramen yugular, y por consiguiente la presencia de síndromes como Villaret, Collet-Sicard y Tapia ${ }^{15}$.

Los estudios imagenológicos para la identificación y caracterización de lesiones en el cóndilo occipital incluyen la tomografía de cráneo y la resonancia magnética cerebral simple y contrastada ${ }^{16,17,18,20}$. A continuación, se describe el caso de una paciente con lesión lítica del cóndilo occipital izquierdo secundaria a mieloma múltiple, quien debutó con cuadro clínico compatible con síndrome del cóndilo occipital izquierdo.

\section{Descripción del caso}

Se trata de una paciente de 67 años previamente sana quien acudió al servicio de urgencias por presentar sintomatología de 2 meses de evolución consistente en dolor en región occipital izquierda y dificultad para la articulación de las palabras, sin disfagia. Al examen físico se encontró aumento del dolor occipital izquierdo con la rotación de la cabeza hacia el lado derecho, y desviación de la lengua hacia el lado izquierdo (Figura 1).

Se obtuvo una tomografía computarizada (TC) de cráneo simple que evidenció lesión lítica en región del cóndilo occipital izquierdo con compromiso del arco posterior de C1 (Figura 2). La imagen de resonancia magnética (IRM) de cerebro con contraste demostró presencia de una lesión tumoral en la base del cráneo que comprometía el hueso occipital izquierdo en su porción basal, el cóndilo izquierdo y el arco posterior de $\mathrm{C} 1$, en relación directa con el conducto del hipogloso izquierdo (Figura 3). Se indicó, por tanto, realización de estudios de extensión en búsqueda de lesión neoplásica primaria, incluyendo TC toracoabdominal contrastado, endoscopia de vías digestivas superiores, colonoscopia, mamografía, antígeno carcinoembrionario y antígeno de cáncer de ovario (CA-125); los cuales resultaron negativos para neoplasias.

Dada la ausencia de diagnóstico histopatológico, y la presencia de signos imagenológicos óseos de inestabilidad craneocervical por compromiso lítico de más del $50 \%$ del cóndilo occipital izquierdo, se decidió realizar resección de la lesión y artrodesis occipitocervical (con tornillos de masa lateral en C3 y C4) por medio de abordaje extremo lateral para cóndilo occipital izquierdo. Una vez expuesto el cóndilo occipital izquierdo, se procedió a realizar fresado del mismo hasta exponer lesión tumoral. La lesión era de localización intradiploica, y de características blandas y violáceas, generando compresión extrínseca del XII par craneal izquierdo. Se resecó $80 \%$ del tumor y se enviaron muestras a patología.

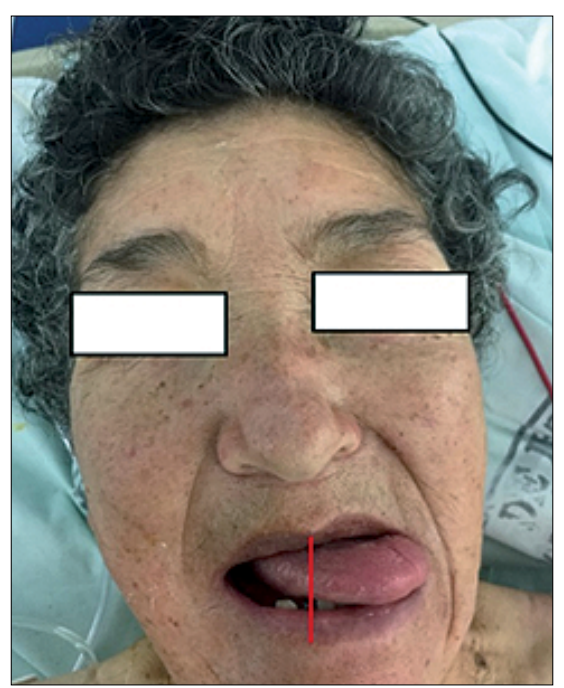

Figura 1. Desviación de lengua hacia el lado izquierdo, secundario a compromiso del XII par craneal izquierdo en el conducto del hipogloso.

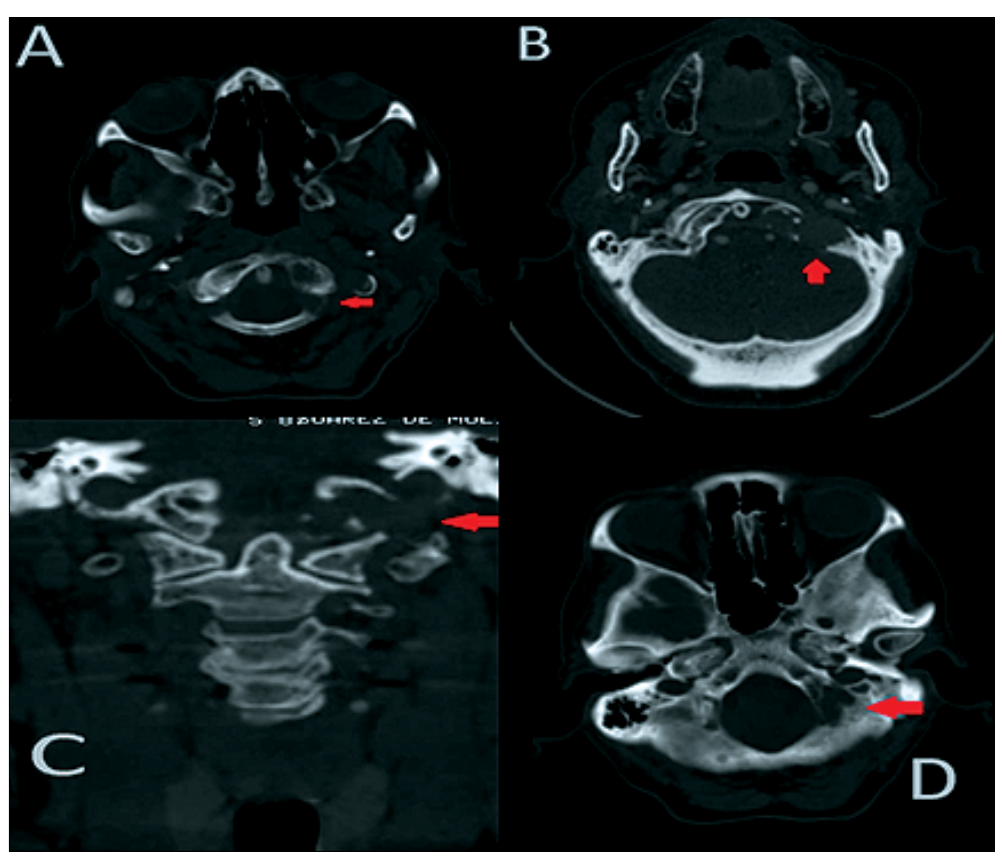

Figura 2. Tomografía computada de cráneo y columna cervical en ventana ósea. Flecha roja señala lesión lítica comprometiendo arco posterior de $C 1(A)$, región condílea izquierda (corte axial: B y coronal: C) y conducto del hipogloso izquierdo (D). 


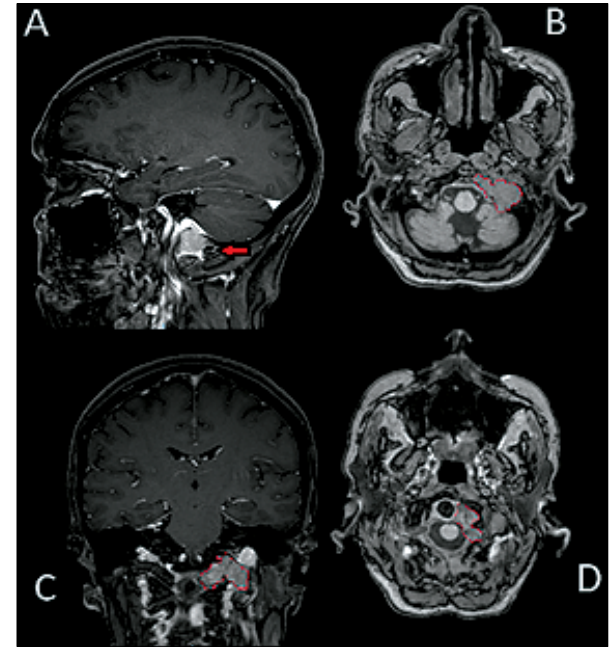

Figura 3. Resonancia cerebral. A: T1 contrastado, corte sagital, flecha roja señala lesión que capta contraste en región condílea izquierda. B, C y D: T1 simple, reborde rojo delimita lesión isointensa infiltrante en región del cóndilo occipital izquierdo (corte axial: B y coronal: C) y en el conducto del hipogloso izquierdo (corte axial: D).

Ya que paciente presentaba también signos de inestabilidad mecánica en articulación occipito-cervical se procedió a realización de fijación posterior occipito cervical (Figura 4).

El estudio histopatológico de la lesión dictaminó infiltración ósea por neoplasia de células plasmáticas (mieloma múltiple), por lo cual fue direccionada al servicio de hematología oncológica. La paciente asistió a cita de control por neurocirugía a los 2 meses luego de la intervención, negando cefalea y disfagia, con evidente mejoría de la paresia del XII par craneal izquierdo.

\section{Discusión}

El síndrome del cóndilo occipital fue reportado por primera vez por Greenberg en 1981. Fue descrito como la aparición de dolor occipital unilateral exacerbado con la rotación de la cabeza hacia el lado contralateral, acompañado de paresia del XII par craneal ipsilateral14,17. Los pacientes frecuentemente refieren dificultad para la articulación de las palabras, disfagia y desviación ipsilateral de la lengua $a^{3,4,5,6,7,8,9,10,13,17}$. La paresia del XII par craneal está comúnmente asociada a atrapamiento extrínseco de dicho par craneal por una lesión tumoral en el conducto del hipogloso $0^{3,6,7,8,10}$. Debido a la vecindad del cóndilo con el foramen yugular, puede cursar con compromiso de los pares craneales IX a XI, generando presencia de síndromes como Villaret, Collet-Sicard y Tapia (Tabla 1).

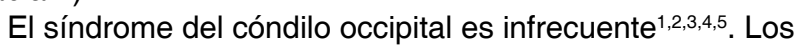
tumores primarios más frecuentemente asociados son el cáncer de mama (55\%), el cáncer de pulmón (14\%), el cáncer de próstata (6\%), el linfoma maligno $(5 \%)$, entre otros $(20 \%)^{12,17}$. El mecanismo más probable de diseminación hacia la base del cráneo es por vía hematógena a través de plexos venosos y vasos transoseos ${ }^{5}$. Otras patologías que pueden afectar el

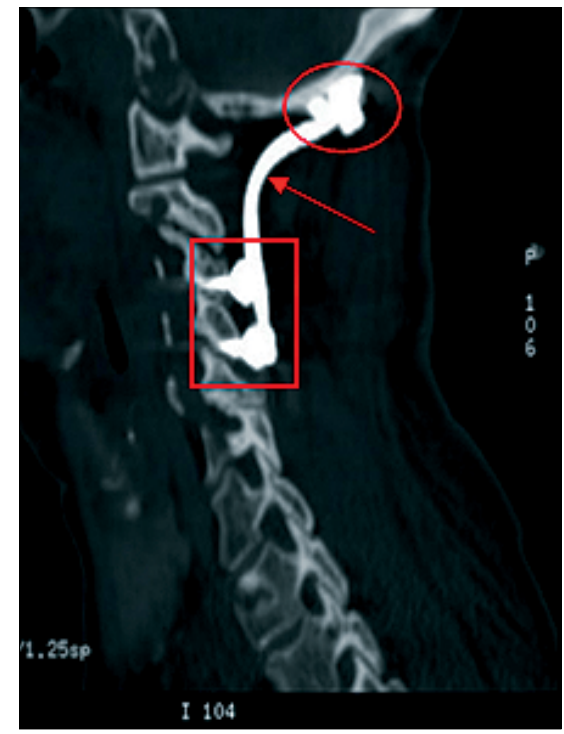

Figura 4. Radiografía de columna cervical, vista lateral. Fijación occipito-cervical con placa occipital (Círculo), barra (flecha) y tornillos en masas laterales cervicales de C3 y C4 (cuadrado).

nervio hipogloso en su porción condilar son traumas, enfermedades inflamatorias y lesiones post-radiación ${ }^{8}$.

Reconocer el síndrome del cóndilo tiene un valor de gran importancia semiológica, ya que su identificación facilita al neurocirujano dar un enfoque diagnóstico topográfico de la lesión. En algunas ocasiones el síndrome del cóndilo puede ser la primera manifestación de una enfermedad oncológica. En el caso de nuestro paciente se documentó compromiso lítico del cóndilo occipital izquierdo por mieloma múltiple, con compresión extrínseca del XII par craneal ipsilateral durante su paso por el conducto del hipogloso.

Para el enfoque diagnóstico, lo primero es solicitar una TC de cráneo simple, la cual permite adecuada visualización de las estructuras óseas de la base del cráneo. Sin embargo, no muestra claramente límites y grados de invasión en tejidos blandos y duramadre en caso de lesiones tumorales ${ }^{5,12,18}$. Si se evidencia o persiste la sospecha compromiso del cóndilo o base del cráneo, se deben complementar estudios con IRM encefálica simple y con contraste, ya que permite apreciar la fosa posterior y sus componentes intraóseos con mayor detalle $^{6,12,20}$. En el mieloma múltiple, como en los plasmocitomas solitarios, se pueden apreciar lesiones líticas que generalmente afectan el clivus y el ápice petroso adyacente en la base del cráneo. Los hallazgos típicos del mieloma son isointensos con la sustancia gris en las imágenes potenciadas de T1, hiperintensas en las imágenes potenciadas de T2 y presentan ávida captación de gadolinio ${ }^{6,12,18}$. La IRM encefálica con administración de gadolinio es el mejor método para detectar metástasis de cráneo-hueso, con una sensibilidad del $82 \%$ para diagnóstico de lesiones líticas ${ }^{5,16,18}$. Otros estudios imagenológicos que se emplean para estudio de enfermedad metastásica en la base del cráneo incluyen la gammagrafía ósea y la tomografía por emisión de positrones $(\mathrm{PET})^{16,18}$.

Si se evidencian imágenes sugestivas de compromiso 


\begin{tabular}{|c|c|c|}
\hline Síndrome & Síntomas y signos & Estructuras involucradas \\
\hline Villaret & $\begin{array}{l}\text { Dependiendo de la extensión de la lesión, se puede apreciar: } \\
\text { - Limitación para la elevación del hombro } \\
\text { - Desviación y atrofia de la lengua } \\
\text { - Cambios en el tono de la voz } \\
\text { - Pérdida del gusto en el tercio posterior de la lengua } \\
\text { - Disfagia } \\
\text { - Cambios sensitivos en el paladar } \\
\text { - Ptosis palpebral parcial y cambios en la sudoración de la } \\
\text { cara }\end{array}$ & $\begin{array}{l}\text { - Foramen yugular: Pares craneales IX, X, XI } \\
\text { - Conducto del hipogloso: Par craneal XII } \\
\text { - Cadena simpática cervical }\end{array}$ \\
\hline Collet-Sicard & $\begin{array}{l}\text { Dependiendo de la extensión de la lesión, se puede apreciar: } \\
\text { - Limitación para la elevación del hombro } \\
\text { - Desviación de la lengua y atrofia } \\
\text { - Cambios en el tono de la voz } \\
\text { - Pérdida del gusto en el tercio posterior de la lengua } \\
\text { - Disfagia } \\
\text { - Cambios sensitivos en el paladar }\end{array}$ & $\begin{array}{l}\text { Foramen yugular: Pares craneales IX, X, XI } \\
\text { - Conducto del hipogloso: Par craneal XII }\end{array}$ \\
\hline Tapia & - Parálisis de la lengua y cuerda vocal ipsilateral & $\begin{array}{l}\text { - Tronco encefálico (núcleo ambiguo, } \\
\text { núcleo del hipogloso y tracto piramidal) o de } \\
\text { origen periférico, con afección de los pares } \\
\text { craneales X y XII }\end{array}$ \\
\hline $\begin{array}{l}\text { Síndrome del } \\
\text { cóndilo occipital }\end{array}$ & $\begin{array}{l}\text { - Presencia de dolor en región occipital ipsilateral que } \\
\text { empeora con la rotación de la cabeza hacia el lado contrario } \\
\text { - Parálisis ipsilateral de la lengua }\end{array}$ & - Conducto del hipogloso: Par craneal XII \\
\hline
\end{tabular}

tumoral de la base del cráneo, incluyendo la región condílea occipital, el siguiente paso es descartar la presencia de un tumor primario sistémico. Para ello se realizan estudios de extensión, dentro de los cuales se incluyen TC toracoabdominal contrastada, mamografía, ecografía testicular, endoscopia de vías digestivas superiores, colonoscopia y antígenos tumorales sanguíneos ${ }^{5,14}$. En nuestra paciente los exámenes de extensión fallaron en demostrar presencia de lesión neoplásica primaria.

El diagnóstico histopatológico definitivo de la lesión es fundamental para dirigir su tratamiento. En la mayoría de casos el diagnóstico se obtiene a través de biopsia abierta o percutánea de la lesión ${ }^{2}$. El manejo médico complementario (radioterapia, quimioterapia, antibioticoterapia, entre otros) suele ser suficiente para aliviar y mejorar los síntomas ${ }^{2,21}$. En caso de lesiones neoplásicas con alto potencial invasor a tejidos aledaños se recomienda resección. En nuestro caso, la paciente presentaba inestabilidad biomecánica craneocervical por compromiso tumoral de más del $50 \%$ de la totalidad del cóndilo occipital izquierdo, por lo que fue llevada a cirugía para resección de lesión y artrodesis occipito-cervical. El abordaje extremo lateral para cóndilo occipital izquierdo favoreció la resección de la lesión condilea; permitió visualización directa del XII par craneal izquierdo emergiendo por el conducto del hipogloso, disminuyendo consecuentemente el riesgo de lesión neurológica iatrogénica; y generó exposición adecuada de estructuras óseas cervicales posteriores para realizar artrodesis occipitocervical posterior desde el occipucio hasta las masas laterales de C4. En nuestra experiencia, el manejo quirúrgico empleado fue seguro, permitió la toma de suficiente material para el estudio histopatológico, brindar estabilidad biomecánica craneocervical y adicionalmente mejoro significativamente los síntomas de dolor y paresia del XII par craneano de la paciente.

\section{Conclusión}

A pesar de que el síndrome del cóndilo occipital es una manifestación clínica poco frecuente, su presencia es altamente valiosa desde el punto de vista semiológico, dado que en la mayoría de los casos se asocia a enfermedad tumoral secundaria en la base del cráneo. Por tanto, es fundamental reconocer este síndrome para realizar un apropiado enfoque diagnóstico y terapéutico de la lesión.

\section{Conflicto de interes}

Este artículo no tiene conflicto de intereses.

\section{Referencias}

1. Pasricha R, Mohanty PP, Madan RC, Datta NR. Occipital condyle metastasis: An unusual clinical presentation in carcinoma of the lung. Radiography. 2005;11(1):41-3.

2. Tomazic P V, Ropposch T, Nemetz U, Walch C. Breast Cancer Metastases of the Left Occipital Condyle Diagnosed through Extended Mastoidectomy: Case Report. Skull Base Reports. 2011;1(1):13-6.

3. Neera C, Yogesh P, Vinod P, A KG. Occipital condyle syndrome in a young male: a rare presentation of cranio-vertebral tuberculosis. J Clin Diagn Res. 2014;8(11):MD01-3.

4. Orliac C, Menjot de Champfleur S, Ducros A, Menjot de Champfleur N. Occipital neuralgia heralding occipital condyle syndro- 
me revealing vesical leiomyosarcoma skull base metastasis. J Neuroradiol. 2015;42(6):368-70.

5. Rodríguez-Pardo J, Lara-Lara M, Sanz-Cuesta BE, Fuentes B, Díez-Tejedor E. Occipital Condyle Syndrome: A Red Flag for Malignancy. Comprehensive Literature Review and New Case Report. Headache. 2017;57(5):699-708.

6. Capobianco DJ, Brazis PW, Rubino FA, Dalton JN. Occipital condyle syndrome. Headache. 2002;42(2):142-6.

7. Martìnez Salamanca JI, Murrieta C, Jara J, Munoz-Blanco JL, Alvarez F, De Villoria JG, et al. Occipital condyle syndrome guiding diagnosis to metastatic prostate cancer. Int $\mathrm{J}$ Urol. 2006;13(7):1022-4.

8. Saraswat MK, Perera RW, Renwick I, Zuromskis T, Singh V, Jones E. Occipital condyle syndrome: self diagnosed. 2018;(fig 1):8-13.

9. Krishnan P, Das S, Salunke P. Occipital condyle syndrome due to tuberculosis: An uncommon cause unearthed by an unconventional approach. Neurol India. 2018;66(2):551-553. doi:10.4103/0028-3886.227324

10. García-Madrona S, Corral-Corral Í. Síndrome del cóndilo occipital como primera manifestación de un carcinoma hepatocelular metastásico . Presentación de dos casos. 2018;66(5):154-6.

11. Abdullah Z, Darrad M, Pathak S. Atrophy of the tongue as the presenting feature of metastatic prostate cancer. Int Neurourol J. 2011;15(3):176-8.

12. Mitsuya $K$, Nakasu $Y$, Horiguchi $S$, Harada $H$, Nishimura $T$, Yuen S, et al. Metastatic skull tumors: MRI features and a new conventional classification. J Neurooncol. 2011;104(1):239-45.

13. Kohli PS, Gandotra P. Small cell bronchogenic carcinoma presenting as collet-siccard syndrome. Indian J Otolaryngol Head
Neck Surg. 2011;63(July):S29-32.

14. Moeller JJ, Shivakumar S, Davis M, Maxner CE. Occipital condyle syndrome as the first sign of metastatic cancer. Can J Neurol Sci. 2007;34(4):456-9.

15. Antoine JC, Camdessanché JP. Peripheral nervous system involvement in patients with cancer [published correction appears in Lancet Neurol. 2007 May;6(5):389]. Lancet Neurol. 2007;6(1):7586. doi:10.1016/S1474-4422(06)70679-2

16. Borges A., 2018. Skull Base Bone Lesions II: Benign and Malignant Tumors. Skull Base Imaging, Chapter 16, 351-37. St. Louis, Missouri: Elsevier. Recuperado de: https://www-clinicalkey-es. ez.unisabana.edu.co/\#!/content/book/3-s2.0-B9780323485630 000167 ?scrollTo=\%23hl0000665

17. Martín Asenjo M, et al. Síndrome del cóndilo occipital como manifestación primera de una neoplasia de recto. Neurología. 2017. https://doi.org/10.1016/j.nrl.2018.04.009

18. Bag AK, Chapman PR. Neuroimaging: intrinsic lesions of the central skull base region. Semin Ultrasound CT MR. 2013;34(5):412-435. doi:10.1053/j.sult.2013.08.004

19. Newman NB, Puthenpura V, Mischell S, Ferreira G. Hypoglossal Nerve Mononeuropathy as the First Presenting Symptom of Progressing Multiple Myeloma. World J Oncol. 2017;8(1):15-17. doi:10.14740/wjon1000w

20. Sekhar LN, Ramanathan D. Evolution of far lateral and extreme lateral approaches to the skull base. World Neurosurg. 2012;77(5-6):617-618. doi:10.1016/j.wneu.2011.05.006

21. Tuchman A, Yu C, Chang EL, Kim PE, Rusch MC, Apuzzo ML. Radiosurgery for metastatic disease at the craniocervical junction. World Neurosurg. 2014;82(6):1331-1336. doi:10.1016/j. wneu.2014.08.061 